\title{
Model Reference Self-Adaptive Control Systems based on Single Neurons
}

\author{
Xiaobin Liu ${ }^{1,}$, Mengda $\mathrm{Li}^{2, \mathrm{~b}}$ \\ ${ }^{1}$ EE Dept. Changzhou Institute of Technology, Jiangsu Changzhou 213032, China \\ ${ }^{2}$ School of Electrical Engineering, Shanghai Dianji University, Shanghai 201306, China \\ a|xblmdlym@126.com, blmdlxblym@126.com
}

Keywords: Model reference, Vector control, Single neuron.

\begin{abstract}
This paper presents a model based on single neuron for adaptive control. For the weakness of traditional PID controllers and complex neural networks, based on the model reference adaptive control, this text using a single neuron instead of a complex neural network, choose the linear function as the reference model, and take the velocity change into the error function, and built the control system with the structure of magnetic chain open-loop and rotate speed close-loop. And then, the control system simulation model based on this control method was established by asynchronous motor, and applied the TMS320 series DSP to set up experimental control system. The simulation and experimental results show that the controller robust is strong, with adaptive characteristics of time-varying parameters and load.
\end{abstract}

\section{The Introduction}

Excitation component and torque component of ac motor stator current exists strong coupling, although adopt vector control, through the magnetic field orientation, can make the magnetic flux and torque to obtain the approximate decoupling, but as a typical nonlinear system of multiple input multiple output, the nonlinear characteristic caused by not only the electromagnetic relation of nonlinear, but also nonlinear characteristics of the hardware conditions, such as the influence of dead zone characteristics, pressure drop characteristics of power semiconductor devices etc. Especially in the fields such as the external environment complex situation, the analysis of the nonlinear characteristics make asynchronous motor system control is extremely complex, this requires that we study the new control method, using advanced control theory for accurate control of its high performance.

The PID controller[1] in the traditional vector control system cannot adjust the control parameters timely, and the neural network controller[2][3] is complicated and difficult to realize. According to this characteristic, the adaptive control system of single neuron is proposed in this paper, this methods used the reference model, established single neuron model reference adaptive controller to instead of traditional PID controller, and applied to induction motor vector control system. Simulation and experimental results show that the single neuron controller robustness and adaptability is very strong, it can greatly improve the performance of the asynchronous motor.

\section{Control Strategy and Control Method}

The single-neuron control structure is shown in figure 1. 


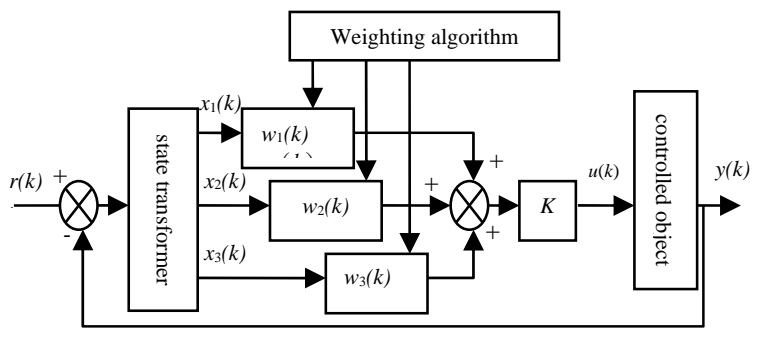

Fig.1 A single neuron control chart

So we take the input of the single neuron respective as

$$
\begin{gathered}
x_{1}(k)=e(k)=r(k)-y(k) \\
x_{2}(k)=T \cdot \sum \Delta e(k) \\
x_{3}(k)=[e(k)-2 e(k-1)+e(k-2)] / T
\end{gathered}
$$

This three quantities are systemic error, error accumulation and first-order difference; Neurons search by correlation, and compute output signals $u(k)$,

$$
u(k)=u(k-1)+K \sum w_{i}(k) \cdot x_{i}(k)
$$

$k$ is the amplification factor of adjustment, $w_{i}(k)$ is the weight coefficient, the weight learning algorithm adopts the supervised Hebb learning rule.

$$
w_{i}(k)=w_{i}(k-1)+\eta_{i} e(k) u(k) x_{i}(k)
$$

The traditional error function is $\left(y_{m}-y_{e}\right)^{2} / 2$, If we learn from this error function, we can't immediately get the output value of the asynchronous machine. So, in this paper ,the change trend of the speed, also the output of the motor, is considered as the error function, and the error function is improvement for

$$
\left(y_{m}-y_{e}\right)^{2} / 2+k_{d}\left(\dot{y}_{m}-\dot{y}_{r}\right)^{2} / 2
$$

$k_{d}$ is the adjustable differential constant, If we use $\omega^{*}$ for a given speed, then the error function expression is converted to

$$
\left(y_{m}-y_{e}\right)^{2} / 2+k_{d}\left(\dot{y}_{m}-\dot{y}_{r}\right)^{2} / 2
$$

In the model reference adaptive control structure, the single neuron is used as the controller, which makes the single neuron model reference adaptive controller, as shown in figure 2. To output $y_{m}$ as the ideal output from the reference model, The error $e=y_{m}-y_{r}$ are generated when the object is altered by internal or external factors that cause the output $y_{r}$ to deviate from the ideal output $y_{m}$, At this time, adjust the power of the neuron by the fuzzy correction gain method, and the controller output is changed, this moment, let A be in line with B , and the error goes to zero.

\section{System modeling}

The model reference controller subsystem model is shown in figure 2 using the above control strategy.

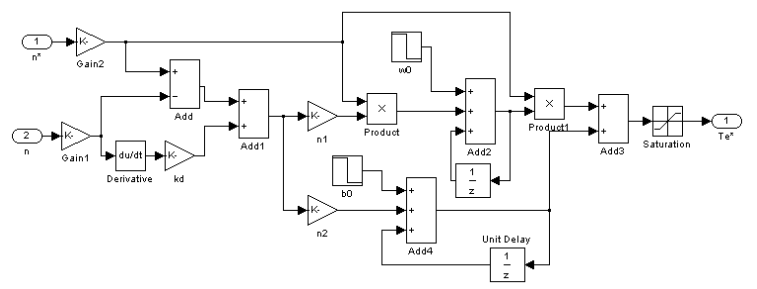

Fig.2 The single neuron model reference controller subsystem

We apply the controller to the asynchronous motor vector control system. The model of single neuron direct model reference adaptive control system for asynchronous motor is shown in figure 3. The control system consists mainly of single neuron controller module, coordinate transform module, current loop PWM module, asynchronous motor module and magnetic chain observation module. 


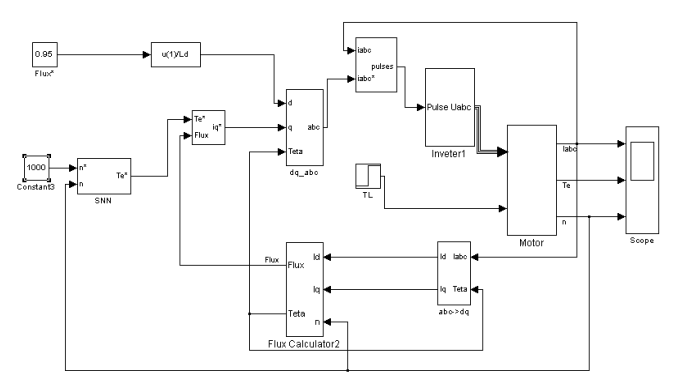

Fig.3 System simulation model of the asynchronous motor single neuron adaptive control

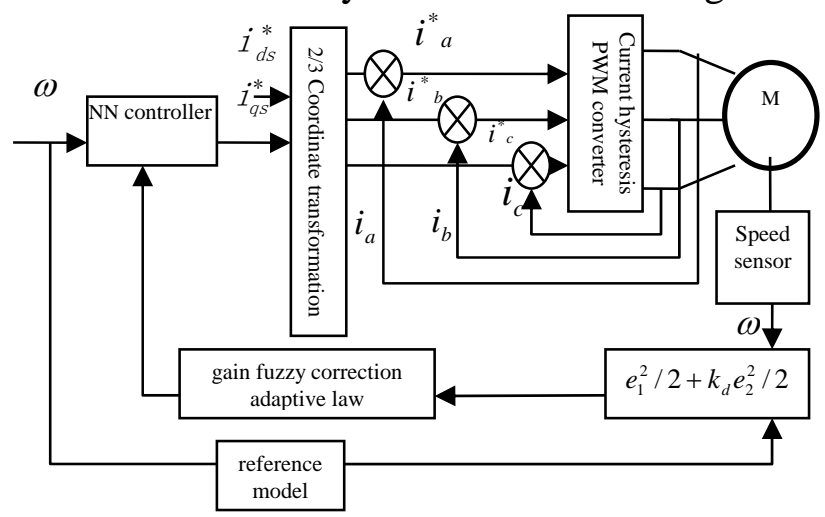

Fig.4 Vector control system of asynchronous motor single-neuron model reference adaptive controller

Vector control system adopts the control structure of magnetic chain open-loop control and speed closed-loop control. The motor ontology in the Static coordinate system is chosen as the reference model. Figure 4 shows the structure diagram for this vector control system.

In this control system, when there is an error between the output of the reference model and the output of the asynchronous motor, we needed a certain adaptive control laws to adjust the weights value and the thresholds value of single neuron. This operation makes the error function $E=e_{1}^{2} / 2+k_{d} e_{2}^{2} / 2$ approach zero, Among them, $e_{1}=\omega^{*}-\omega, e_{2}=\dot{\omega}^{*}-\dot{\omega}$. This method enables the motor speed output tracking reference model output.

\section{System Simulation and Experimental Verification}

Select asynchronous motor parameters: $\tau_{e n}=10 \mathrm{~N} \cdot \mathrm{m}, \mathrm{P}=1.5 \mathrm{KW}, R_{r}=2.5 \Omega, R_{s}=3.9 \Omega$, $L_{s}=0.5 \mathrm{H}, \quad L_{r}=0.5 \mathrm{H}, \quad L_{m}=0.6 \mathrm{H}, \quad n_{p}=2, \quad \mathrm{~J}=0.002 \mathrm{Kg} \cdot \mathrm{m}^{2}$, System initial parameter : $\eta_{1}=0.03, \eta_{2}=0.03$ 。

Let's assuming that the expect speed is the step function of 1,000 r/min, Figure 5 shows the speed curve of using the single neuron controller, Figure 6 shows the speed curve of using the traditional PID controller. We can see from the graph that the system speed of using single neuron controller increases faster and no overshoot. In addition, the traditional PID controller needs to adjust three parameters at the same time, in this paper, the single neuron controller only needs to adjust one parameter $k_{d}$ to get better control quality.

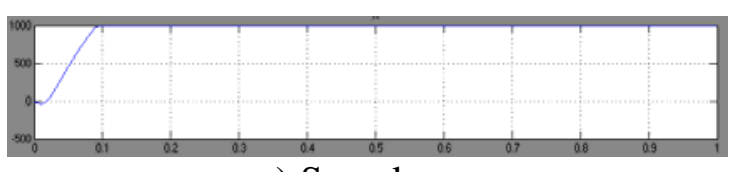

a) Speed curve

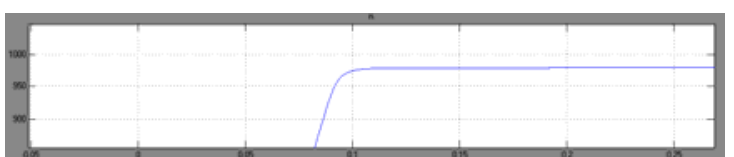

b) Local amplification figure

Fig. 5 The motor speed curve of using single neuron model reference controller 


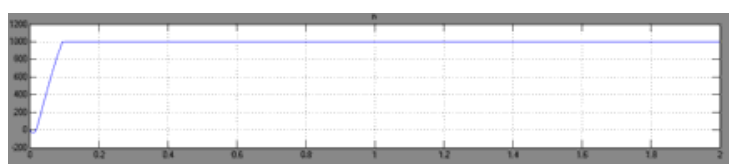

a) Speed curve

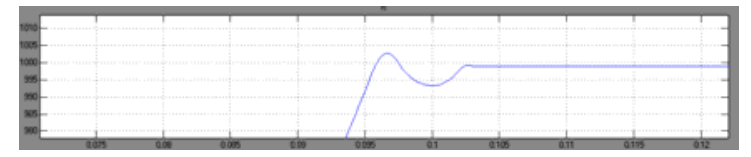

b) Local amplification figure

Fig.6 Speed curve of using traditional PID controller

Figure 7 is the speed tracking curve for asynchronous motors. The speed response curve is shown in figure 8, with a load of $10 \mathrm{~N} \cdot \mathrm{m}$ at the start, and $15 \mathrm{~N} \cdot \mathrm{m}$ at one second, We can see that the speed has small fluctuation and can recover quickly, indicating that the controller has strong load anti-interference ability. Figure 9 shows the rotor speed curve before and after the rotor resistance change respectively. Increase the resistance of the rotor by $50 \%$, and the response speed is almost unchanged. It is shown that the controller has strong robustness against the change of the rotor resistance of the motor.

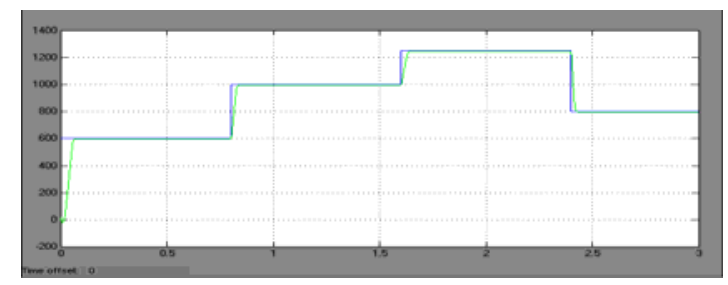

Fig.7 Speed tracking curve

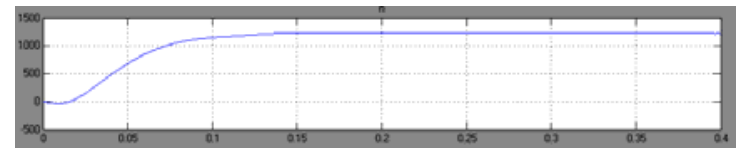

a) Before the change3.75 $\Omega$

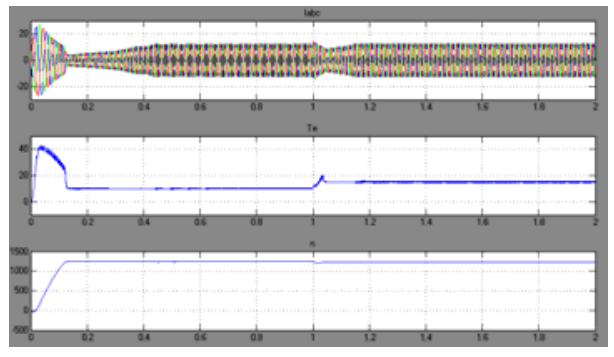

Fig.8 the stator current,torque, speed curve when the load changes

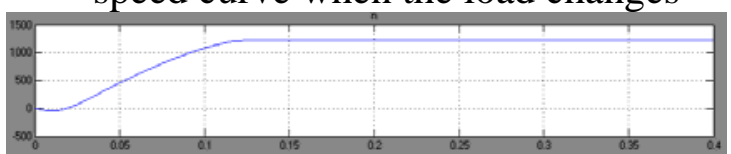

b) After the change2.5 $\Omega$

Fig.9 The speed response curve of rotor resistance changes before and after

Based on high-speed digital processor TMS320LF2407A of TI company, the experimental control system was built. This system consists of the main circuit, the control circuit and the protective circuit. The hardware block diagram is shown in figure 10, The hardware system consists of the rectifier filter module, the inverter module, the IPM protection module, the limited-flow start-up module, the voltage detection module, the driver module, etc. DSP is used to implement the control algorithm of single neuron controller and the conventional PID control algorithm, and to detect, transform and data processing of signals such as speed, current, etc. The PC part adopts the vc ++ to write the communication interface, which is mainly responsible for the functions of speed, frequency, and magnetic flux, and the fault display and processing for the speed control system.

The experiment was: Set motor speed of $1500 \mathrm{r} / \mathrm{min}$, the experiment time was $0.2 \mathrm{~s}$, load is suddenly added at $\mathrm{t}=0.08 \mathrm{~s}$, The speed waveform of using the conventional PID controller and using the single-neuron model were observed respectively, as shown in figure 11 , It is obvious that the traditional PID has a higher rotational speed overshoot and a longer adjustment time. But under the control of single neuron model reference adaptive, there are smaller speed overshoot and a shorter adjustment time, the rotate speed faster recovery when the load changes. Experiments show that: The single neuron controller has better adaptability and robustness, which can improve the performance of asynchronous motor vector control. 


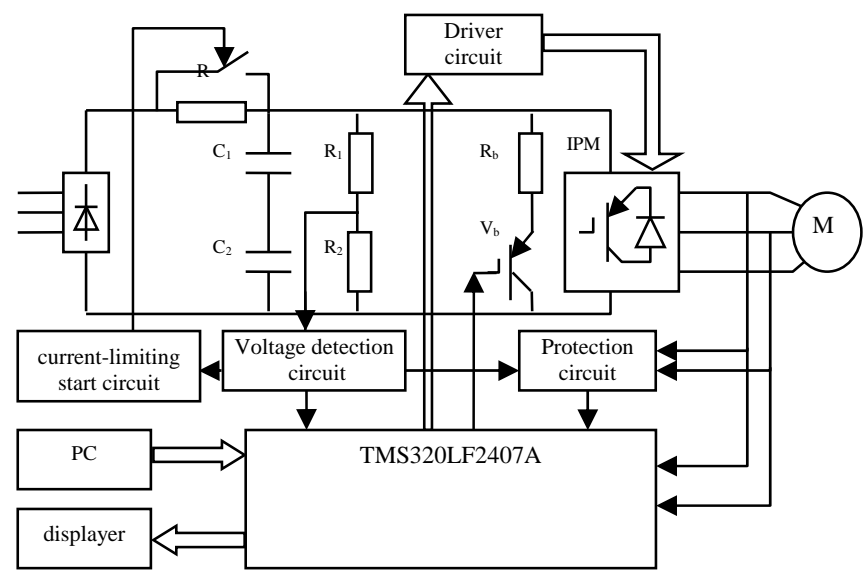

Fig.10 Hardware control block diagram

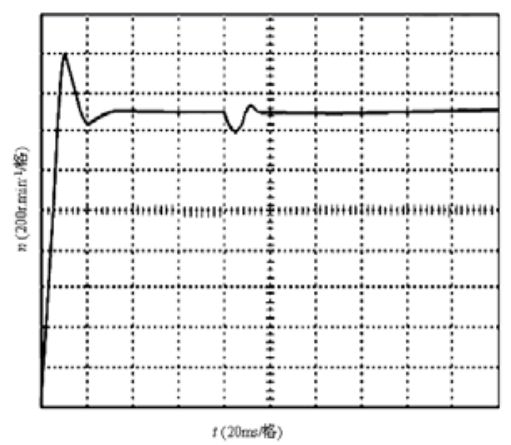

a) The traditional PID controller

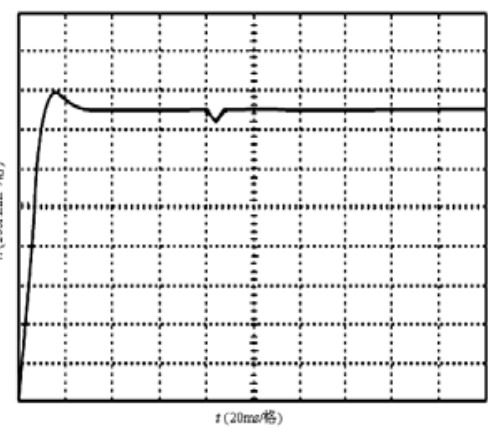

b) Single neuron controller

Fig.11 Speed response waveform

\section{Conclusion}

This paper builds a model reference adaptive controller based on single neuron. Through simulation and experimental verification on asynchronous motor, the system has good dynamic static performance. Based on single neuron parameters online adjustment, we can make the controller have a strong adaptive ability and robustness for a given value changes, load disturbance and parameter changes.

\section{References}

[1]. Yangzhi, Zhuhaifeng, Huangyihua. Control and Instruments in Chemical Industry [J]. Chemical automation and instrumentation. 2005, 32 (2): 127.

[2]. Li Zulinl, ZhouDong fang. Contrivanee of Intelligent DC Speed Regulating System of Drilling Rig Driving Motor[J]. Electrotechnology. 2003.9:54-55.

[3]. Wang Xiaodong,Chen Boshi. Analysis on Stable Ability of Single Neuron Adaptive Control for Drive Systems[J]. Electric drive 2003.1:25-29.

[4]. Zhang Hui, Yu Haisheng, Wu Herong in Jinpeng. Research of asynchronous motor drive based on fuzzy controller [J]. Journal of Qiingdao University (E \& T). 2014,29 (3): 1-6.

[5]. JIA Li,TAO Pengye,QIU mingsen. Adaptive Single-Neuron Control System Design Based on Fuzzy Neural Network Model. ZJournal of East China Universitv of Science and Technoloev (Natural Science Edition)[J]. 2008, 34(2):135-139,143.

[6]. YANG youlin, WU qinghui. Adaptive Speed Control of Asynchronous Motor Based on Single Neuron PID[J]. Electrical Automation. 2013, 35(5):36-38. 